\title{
Response of benthic soluble reactive phosphorus transfer rates to step changes in flow velocity
}

\author{
Tetsunori Inoue • Yoshiyuki Nakamura
}

Received: 11 July 2011 / Accepted: 21 August 2012 / Published online: 12 September 2012

(C) The Author(s) 2012. This article is published with open access at Springerlink.com

\begin{abstract}
Purpose The purpose of this study was to investigate the responses of the benthic soluble reactive phosphorus (SRP) transfer rate to step changes in the flow velocity of the overlying water using laboratory experiments and a nonsteady-state numerical model.

Materials and methods Laboratory experiments were conducted using a rectangular recirculating flume. After preincubation of sediments in a cavity of the experimental flume for 2 days, the step responses of the SRP transfer rate to sudden increases in the flow velocity were examined under anaerobic conditions. The benthic SRP transfer rates were obtained from the rate of increase in the SRP concentration of the overlying water. We also analysed the response using a newly constructed numerical model that consists of a one-dimensional diffusion model of the diffusive boundary layer (DBL) and a biochemical model of the sediment, in which oxygen, SRP, ferrous iron and nitrate were model variables. The non-steady-state calculation was performed to reproduce the experiments after the step change in the flow velocity.

Results and discussion The experiments revealed a rapid increase in the SRP concentration in the overlying water that continued for approximately $5 \mathrm{~min}$ after the step change in flow velocity and was followed by a lower, steady
\end{abstract}

Responsible editor: Geraldene Wharton

T. Inoue $(\bowtie)$

Coastal and Estuarine Environment Field, Port and Airport

Research Institute,

3-1-1, Nagase,

Yokosuka 239-0826, Japan

e-mail: inoue-t@ipc.pari.go.jp

Y. Nakamura

Port and Airport Research Institute,

3-1-1, Nagase,

Yokosuka 239-0826, Japan increase in the SRP concentration. The model analyses also demonstrated that a step increase in flow velocity leads to a drastic enhancement of the SRP transfer rate within a few minutes. The abrupt increase in the transfer rate was due to the rapid transport of SRP that had accumulated in the DBL and to enhanced diffusion caused by a temporal increase in the SRP concentration gradient in the DBL. The modelled results for the SRP transfer rate were in agreement with the experimental results.

Conclusions The temporal increase in SRP transfer was due to rapid SRP transport caused by intensified diffusion following a decrease in DBL thickness. The model-based results for the response of the SRP transfer rate were in agreement with the experimental results. Therefore, our model can simulate the response of the SRP concentration profile near the sediment-water interface to temporal variations in flow velocity.

Keywords Benthic phosphate transfer . Diffusive boundary layer · Flow velocity . Non-steady-state process

\section{Introduction}

Because phosphorus $(\mathrm{P})$ frequently acts as a limiting parameter in the outbreak of algal blooms or red tide, a quantitative understanding of its cycle is required for the environmental management of eutrophic shallow lakes and lagoons (e.g. Gomez et al. 1999). Benthic P transfer is one of the most important factors that controls $\mathrm{P}$ balance in a water column, and short-term P cycling in the sediment is mainly dependent on adsorption to and desorption from iron hydroxide. This process is dependent on several factors, including oxidation-reduction potential and $\mathrm{pH}$ (e.g. McQueen et al. 1986; Gunnars and Blomqvist 1997; Boers et al. 1998). In practice, benthic soluble reactive phosphorus 
(SRP) release is enhanced under anoxic conditions, in which stratification is often observed. Vertical disturbances cause destratification and the supply of SRP from the lower to the surface water layer, consequently inducing algal blooms and other negative impacts. The process is usually accompanied by enhanced flow velocity in the lower water layer.

By contrast, there are some cases in which enhanced flow in the lower water layer is observed without destratification. For example, in Lake Shinji, a shallow brackish lagoon indirectly connected to the Japan Sea, saline water is intermittently intruded into the bottom to form a thin (typically approximately $50 \mathrm{~cm}$ ) layer with salinity stratification. Because of its thinness, the bottom layer rapidly becomes anoxic, and SRP concentrations also rapidly increase. In parallel, wind-induced non-steady-state wave and current motions in both the surface and bottom layers are often observed without any destratification (Inoue and Nakamura 2002). Fluid motions of the bottom layer, such as internal seiches, have been reported elsewhere (e.g. Brand et al. 2009). Under these conditions, the non-steady-state SRP transfer process should have a specific influence on the water environment.

Hydrodynamic conditions are generally responsible for material transfer in the diffusive boundary layer (DBL) and are often the rate-limiting factor for material transfer at the sediment-water interface (e.g. Inoue et al. 2000; Boudreau 2001). These hydrodynamic effects have been studied frequently for benthic oxygen transfer (Belanger 1981; Boynton et al. 1981; Jørgensen and Des Marais 1990; Inoue and Nakamura 2009), but only a few reports have addressed $P$ transfer. For example, Fowler et al. (1987) developed a pumping and reservoir system for the circulation of water over intact sediment cores and evaluated the effect of flow velocity on SRP exchange at the sediment-water interface. Nakamura (1994) reported a theoretical study of the effect of flow velocity on SRP release from the sediment in which onedimensional diffusion equations with reaction terms were used. Regarding benthic $\mathrm{P}$ transfer, most previous studies have predicted the quasi-steady-state condition and have focused on processes with relatively long time scales, such as seasonal variations (e.g. Gonsiorczyk et al. 2001). Although there have been some studies of short-time-scale processes (Löfgren and Böstrom 1989; Gunnars and Blomqvist 1997; Spears et al. 2007), most of these studies have exclusively studied the geochemical, and not the hydrodynamic, aspect.

Therefore, the purpose of this study was to quantify the response of the transient benthic SRP release rate to step changes in the flow velocity of the overlying water with a laboratory flume experiment. Moreover, a non-steady-state numerical model consisting of oxygen, SRP, ferrous iron and nitrate reaction terms was also designed, and the calculated results were compared with the laboratory experimental results to quantitatively understand the non-steady-state processes.

\section{Materials and methods}

\subsection{Experimental methods}

In this study, we employed the previously described (Inoue and Nakamura 2009) rectangular recirculating flume, which was $15 \mathrm{~cm}$ in height, $12.5 \mathrm{~cm}$ in width and $250 \mathrm{~cm}$ in length (Fig. 1). Sediment was situated in a cavity that was $10 \mathrm{~cm}$ in depth and $100 \mathrm{~cm}$ in length in the middle section of the flume bed. The flow velocity and water temperature were controlled using a variable pump, flow meter and temperature controller with $\pm 0.1{ }^{\circ} \mathrm{C}$ accuracy (LPA3, ORION, Suzaka, Japan). The water in the flume was sampled from the sampling valve without exposure to air. Oxygen concentration was monitored with an oxygen meter (DO-25A, TOA, Tokyo, Japan) with a probe that was installed at the downstream end of the flume.

The sediment and the overlying water were sampled at an inter-tidal flat that is located in the inner part of Hakata Bay in the western part of Japan. The sampled sediment and water were immediately transported to the laboratory, and the sediment was placed in the cavity of the experimental flume as described earlier. The recirculating water in the flume had previously been filtered through a fibre glass filter (Whatman GF/C) and was maintained at $25{ }^{\circ} \mathrm{C}$. For the pre-incubation, the overlying water was deoxygenated by bubbling with $\mathrm{N}_{2}$ gas for $3 \mathrm{~h}$ and recirculated with a pump at a flow velocity of $1.7 \mathrm{cms}^{-1}$ for 2 days. The oxygen concentration in the overlying water was assessed to confirm that a completely anoxic condition was achieved. Before initiating the experiment, tweezers were used to remove as many benthic organisms as possible.

At the beginning of this study, the flow velocity was raised instantaneously from 1.7 to $5.0 \mathrm{cms}^{-1}$, and it defined the start of the experiment $(t=0)$. This condition was maintained for 1 day and defined as experiment 1 . Aliquots of the overlying water were sampled from the sampling valve for the chemical analyses described later. The sampling interval was between 3 and $10 \mathrm{~min}$ for the first $20 \mathrm{~min}$ and for several hours thereafter. Aliquots of the samples were used for turbidity measurements. The remaining samples were

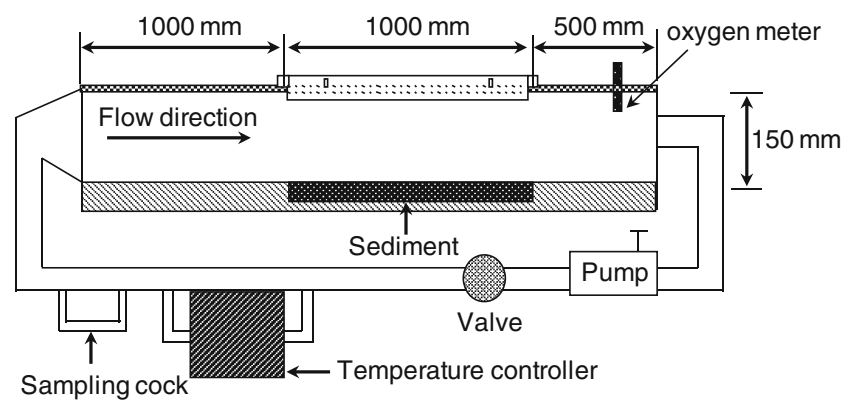

Fig. 1 Schematic view of the rectangular recirculation flume system 
filtered through a Whatman GF/F fibre glass filter and used for the analysis of SRP and ferrous iron. After 1 day of incubation with a flow velocity of $5.0 \mathrm{cms}^{-1}$, the flow velocity was changed from 5.0 to $10.0 \mathrm{cms}^{-1}$, and sampling was performed in the same way as stated for the previous day (defined as experiment 2). Before and after the entire experiment, the overlying water was sampled, and nitrate concentration was analysed. The flow velocity profiles were measured using a laser Doppler velocimeter (Model 9710, TSI, Shoreview, MN, USA), and the relationship between the cross-sectional mean flow velocity and the friction velocity, $u^{*}$, was obtained based on a logarithmic profile of velocity. The information from $u^{*}$ was used to evaluate the turbulent diffusion coefficient in the model-based analysis described later.

In this experiment, the SRP transfer rates from the sediment were obtained from the rates of increase in the SRP concentration in the overlying water with the following equation:

$V \frac{\mathrm{d} C}{\mathrm{~d} t}=$ Flux $\cdot A+r \cdot V$

where $V$ is the entire volume of recirculating water in the system, $C$ is the SRP concentration, $t$ is time, Flux is the SRP transfer rate from the sediment, $A$ is the surface area of the sediment and $r$ is the volume-specific SRP generation rate in the water.

\subsection{Post-experiment procedures}

After the experiment, known volumes of sediment layers were sampled using end-cut syringes. Some portions of the sediment samples were dried at $60{ }^{\circ} \mathrm{C}$ for 2 days, and porosity was estimated from the weight difference before and after the drying process by considering pore water density. Other samples were used to measure the volumespecific oxygen consumption rate of the sediment, which was determined according to the method of Hosoi et al. (1992). This method involved measuring the rate at which oxygen concentration decreased in a known volume of water that was mixed with a known volume of sediment, and the rate was defined as the total oxygen consumption rate. The rate was also determined for water samples where biological activity was suppressed by the addition of $5 \%$ formalin, and the rate was assumed to be the chemical oxygen consumption rate (Urban-Malinga and Opalinski 1999). Moreover, the difference between the total and chemical oxygen consumption rates was defined as the biological oxygen consumption rate. The rates at which the SRP concentration decreased and increased in a known volume of water that was mixed with a known volume of sediment were also measured to determine the volume-specific SRP adsorption and desorption rates, respectively, with a procedure similar to that employed for determining the volume-specific oxygen consumption rate.

Some of the sediment samples collected with the syringe were centrifuged at 3,000 r.p.m. for $10 \mathrm{~min}$ to separate pore water in $\mathrm{N}_{2}$-filled tubes. The obtained pore water was filtered with a disposable filter with a pore size of $0.45 \mu \mathrm{m}$ (Minisarto SM16555K, Sartorius, Tokyo, Japan) and stored at $-25^{\circ} \mathrm{C}$ in a freezer for subsequent chemical determination of the SRP and ferrous iron concentrations. The SRP, nitrate and ferrous iron concentrations were determined by the ascorbic acid method, cadmium reduction-colorimetric method and phenanthroline method (Clesceri et al. 1998), respectively, with a spectrophotometer (UV-1200, Shimazu, Kyoto, Japan). These parameters were employed in the subsequent analyses and calculations.

\subsection{Numerical model for calculation of non-steady-state SRP release}

The numerical model used here consists of a onedimensional diffusion model of the DBL and a biochemical model of the sediment. This structure follows the original concept of Nakamura (1994), as modified by Inoue et al. (2000). We extended this model through the addition of a non-steady-state term for the study of short-time-scale processes. In this model, oxygen is supplied from the water to the sediment by diffusion through the DBL and is consumed by biochemical reactions in the sediment. SRP in pore water is supplied by desorption from sediment particles and is transported by diffusion to the overlying water. We assume that SRP simultaneously adsorbs to ferric hydroxide produced by the abiotic oxidation of ferrous iron in the aerobic layer (Beutel et al. 2008). Other processes relating to $\mathrm{P}$ cycling in the surface sediment, such as release from microorganisms (e.g. Gächter et al. 1988) and/or adsorption to calcite (e.g. Boström et al. 1988), are not considered in the model because they are relatively slow processes and can be ignored under anoxic conditions.

In the experiment described earlier, the nitrate concentration in the overlying water was high (approximately $200 \mu \mathrm{moll}^{-1}$ ) and was included because of its preventive effect on ferric hydroxide reduction (Löfgren and Böstrom 1989). Assuming that the biological nitrification rate is so slow that it has no effect on short-term SRP dynamics (Beutel 2006), the physico-chemical process for nitrate was formulated simply by considering the diffusion and denitrification processes. According to Sørensen (1982), additions of $0.2 \mathrm{mmoll}^{-1}$ nitrate and nitrite inhibit $\mathrm{Fe}^{3+}$ reduction. In our calculation, SRP desorption from the sediment particles was assumed to occur only when the nitrate concentration was $<0.1 \mathrm{mmoll}^{-1}$. A reasonable variation in this threshold value will not affect the main result of this paper (see the succeeding 
discussion). The model calculation was conducted to follow the experimental procedure as closely as possible, from the experimental setup under aerobic conditions to the end of the pre-incubation under anoxic conditions. The oxidation of ferrous iron under aerobic conditions was estimated by the model to give a reasonable profile of SRP and ferrous iron for the initial condition, which would affect the SRP valance during experiments 1 and 2 .

The diffusion and biochemical reactions in the sediment stated earlier were included in the mass balance equations for oxygen, SRP, ferrous iron and nitrate as follows:

$$
\begin{aligned}
\varphi \frac{\partial C_{\mathrm{O}}}{\partial t} & =\varphi D_{z \mathrm{SO}} \frac{\partial^{2} C_{\mathrm{O}}}{\partial z^{2}}-\frac{1}{4} \varphi k_{\mathrm{OF}} C_{\mathrm{O}} C_{\mathrm{F}}-k_{\mathrm{B}}(\text { for } z<0) \\
\varphi \frac{\partial C_{\mathrm{P}}}{\partial t} & =\varphi D_{z \mathrm{SP}} \frac{\partial^{2} C_{\mathrm{P}}}{\partial z^{2}}-\alpha k_{\mathrm{OF}} C_{\mathrm{O}} C_{\mathrm{F}}-k_{\mathrm{ad}} C_{\mathrm{P}} \\
& -k_{\mathrm{de}}\left\{C_{\mathrm{P}}-C_{\mathrm{P}}(-\infty)\right\}(\text { for } z<0) \\
\varphi \frac{\partial C_{\mathrm{F}}}{\partial t} & =\varphi D_{z \mathrm{SF}} \frac{\partial^{2} C_{\mathrm{F}}}{\partial z^{2}}-\varphi k_{\mathrm{OF}} C_{\mathrm{O}} C_{\mathrm{F}} \\
& -\frac{1}{\alpha} k_{\mathrm{de}}\left\{C_{\mathrm{P}}-C_{\mathrm{P}}(-\infty)\right\}(\text { for } z<0) \\
\varphi \frac{\partial C_{\mathrm{N}}}{\partial t} & =\varphi D_{z \mathrm{SN}} \frac{\partial^{2} C_{\mathrm{N}}}{\partial z^{2}}-k_{\mathrm{denit}}(\text { for } z<0)
\end{aligned}
$$

where $C_{\mathrm{O}}, C_{\mathrm{P}}, C_{\mathrm{F}}$ and $C_{\mathrm{N}}$ are the concentrations of oxygen, $\mathrm{SRP}$, ferrous iron and nitrate, respectively; $D_{z \mathrm{sO}}, D_{z \mathrm{sP}}, D_{z \mathrm{SF}}$ and $D_{z \mathrm{sN}}$ are the apparent diffusion coefficients in the sediment for oxygen, SRP, ferrous iron and nitrate, respectively; $z$ is the vertical axis from the sediment-water interface (positive upward and zero at the sediment-water interface); $\varphi$ is porosity; $k_{\mathrm{OF}}$ is the rate constant for ferrous iron oxygenation; $k_{\mathrm{B}}$ is the biotic oxygen consumption rate; $\alpha$ is the molar ratio of increases in SRP and ferrous iron concentrations during SRP desorption; $k_{\mathrm{ad}}$ is the SRP adsorption rate; $k_{\mathrm{de}}$ is the SRP desorption rate; $k_{\text {denit }}$ is the denitrification rate; and $C_{\mathrm{P}}(-\infty)$ is the SRP concentration in the pore water at $z=-\infty$. In this study, the oxidation of ferrous iron was assumed to represent the chemical oxygen consumption in the sediment, and denitrification was assumed to occur only when the oxygen concentration was below $0.02 \mathrm{mmoll}^{-1}$. The apparent diffusion coefficients, $D_{z \mathrm{~s}}$, were defined as follows:

$D_{z \mathrm{~s}}=\varphi^{m-1} D_{z \mathrm{~m}}($ for $z<0)$

where $D_{z \mathrm{~m}}$ is the molecular diffusion coefficient, which varies depending on the targeting solution or ion (Boudreau 1997), and $m$ is the numerical constant (and was assumed to be 3 given the silty sand texture of the sediment; Ullman and Aller 1982).
In the water column, biochemical reactions are assumed to be negligible, and the formulations are simplified as:

$$
\begin{gathered}
\frac{\partial C_{\mathrm{i}}}{\partial t}=D_{z \mathrm{w}} \frac{\partial^{2} C_{\mathrm{i}}}{\partial z^{2}}(\text { for } \mathrm{z} \geq 0) \\
D_{z \mathrm{w}}=D_{z \mathrm{~m}}+D_{z \mathrm{t}}(\text { for } \mathrm{z} \geq 0)
\end{gathered}
$$

$$
\frac{D_{z t}}{v}=\left(A \frac{z u^{*}}{v}\right)^{n}(\text { for } z \geq 0)
$$

where $C_{\mathrm{i}}$ represents each solution or ion concentration, $D_{z \mathrm{w}}$ is the vertical diffusion coefficient in water, $D_{z \mathrm{t}}$ is the turbulent diffusion coefficient, $\nu$ is the kinematic viscosity, $u^{*}$ is the friction velocity and $A(=0.078)$ and $n(=3)$ are numerical constants (Nakamura and Mikogami 1994). In this model, the effect of flow velocity is represented by $u^{*}$, and an increase in $u^{*}$ leads to an increase in the turbulent diffusion coefficient, resulting in a decrease in the DBL thickness and an increase in the diffusive transfer rate.

The calculation conditions were designed to follow the sampling and experimental procedures. Namely, the nonsteady-state calculation was conducted under anoxic and $1.7 \mathrm{cms}^{-1}$ flow velocity conditions with steady oxic solutions as initial conditions to reproduce the pre-incubation environment. Thereafter, the non-steady-state calculation after the step change in the flow velocity from 1.7 to $5.0 \mathrm{cms}^{-1}$ was conducted to reproduce experiment 1 . The subsequent calculation was also conducted in the same way for experiment 2 , in which the flow velocity was increased from 5.0 to $10.0 \mathrm{cms}^{-1}$. The parameters employed in this calculation are summarized in Table 1 .

Inoue and Nakamura (2011) investigated solute diffusion across a sediment surface and proposed a simple quantification of the enhancement effect for diffusive transfer owing to a bursting phenomenon in the benthic boundary layer. Following their report, we revised the calculation of the fluxes with the enhancement factor, $F$, expressed as follows:

$F=0.021 u^{* 2}-0.181 u^{*}+1.985$

where $u^{*}$ is in $\mathrm{cms}^{-1}$.

\section{Results}

\subsection{Experimental results}

Because the sampling site was strongly affected by river discharge from a neighbouring urban area, the silty sediment associated with this eutrophic site was characterized by a high oxygen demand. No oxygen or ferrous iron was detected in the overlying water throughout the experiment, 
Table 1 Parameters used in the numerical model calculations

\begin{tabular}{|c|c|c|c|}
\hline Parameter & Symbol & Value & Unit \\
\hline Oxygen concentration in the bulk of the water & $C_{\mathrm{O}}(\infty)$ & $0.00 \mathrm{E}+00$ & $\mu \mathrm{moll}^{-1}$ \\
\hline SRP concentration in the bulk of the water & $C_{\mathrm{P}}(\infty)$ & $1.25 \mathrm{E}+00-3.73 \mathrm{E}+00$ & $\mu \mathrm{moll}^{-1}$ \\
\hline Ferrous iron concentration in the bulk of the water & $C_{\mathrm{F}}(\infty)$ & $1.33 \mathrm{E}-01$ & $\mu \mathrm{moll}^{-1}$ \\
\hline Nitrate concentration in the bulk of the water & $C_{\mathrm{N}}(\infty)$ & $2.01 \mathrm{E}+02$ & $\mu \mathrm{moll}{ }^{-1}$ \\
\hline Oxygen concentration in the bulk of the sediment & $C_{\mathrm{O}}(-\infty)$ & $0.00 \mathrm{E}+00$ & $\mu \mathrm{moll}^{-1}$ \\
\hline SRP concentration in the bulk of the sediment & $C_{\mathrm{P}}(-\infty)$ & $4.34 \mathrm{E}+01$ & $\mu \mathrm{moll}^{-1}$ \\
\hline Ferrous iron concentration in the bulk of the sediment & $C_{\mathrm{F}}(-\infty)$ & $2.71 \mathrm{E}+00$ & $\mu \mathrm{moll}{ }^{-1}$ \\
\hline Nitrate concentration in the bulk of the sediment & $C_{\mathrm{N}}(-\infty)$ & $0.00 \mathrm{E}+00$ & $\mu \mathrm{moll}^{-1}$ \\
\hline Molecular diffusion coefficient of oxygen & $D_{z \mathrm{mO}}$ & $2.29 \mathrm{E}-05$ & $\mathrm{~cm}^{2} \mathrm{~s}^{-1}$ \\
\hline Molecular diffusion coefficient of SRP & $D_{z \mathrm{mP}}$ & $6.20 \mathrm{E}-06$ & $\mathrm{~cm}^{2} \mathrm{~s}^{-1}$ \\
\hline Molecular diffusion coefficient of ferrous iron & $D_{z \mathrm{mF}}$ & $7.06 \mathrm{E}-06$ & $\mathrm{~cm}^{2} \mathrm{~s}^{-1}$ \\
\hline Molecular diffusion coefficient of nitrate & $D_{z \mathrm{mN}}$ & $1.92 \mathrm{E}-05$ & $\mathrm{~cm}^{2} \mathrm{~s}^{-1}$ \\
\hline Porosity & $\varphi$ & $6.53 \mathrm{E}-01$ & - \\
\hline Rate constant for ferrous iron oxygenation & $k_{\mathrm{OF}}$ & $3.08 \mathrm{E}+02$ & $\mathrm{~cm}^{3} \mathrm{~mol}^{-1} \mathrm{~s}^{-1}$ \\
\hline Biotic oxygen consumption rate & $k_{\mathrm{B}}$ & $4.00 \mathrm{E}+06$ & $\mu \mathrm{moll}^{-1} \mathrm{~s}^{-1}$ \\
\hline Molar ratio of increases in SRP and ferrous iron concentrations during SRP desorption & $\alpha$ & $1.40 \mathrm{E}+01$ & - \\
\hline SRP adsorption rate & $k_{\mathrm{ad}}$ & $6.62 \mathrm{E}-04$ & $\mathrm{~s}^{-1}$ \\
\hline SRP desorption rate & $k_{\mathrm{de}}$ & $2.10 \mathrm{E}-04$ & $\mathrm{~s}^{-1}$ \\
\hline Denitrification rate $^{\mathrm{a}}$ & $k_{\text {denit }}$ & $1.50 \mathrm{E}-01$ & $\mu \mathrm{moll}^{-1} \mathrm{~s}^{-1}$ \\
\hline
\end{tabular}

SRP soluble reactive phosphorus

${ }^{a}$ The denitrification rate was adjusted to fit the measured benthic nitrate transfer rate as described previously by Fujita et al. (1997), whose study site was near to our sampling site

and the average nitrate concentration during the experiment was $201.4 \mu \mathrm{moll}^{-1}$, with a slight tendency to decrease over time. A significant increase in turbidity was not observed (data not shown). The SRP concentration in the overlying water continuously increased as a result of continuous SRP release from the sediment throughout the experiment (Fig. 2). In all of the experiments, a rapid increase in the SRP concentration in the overlying water was observed for approximately $5 \mathrm{~min}$ after the step change in the flow velocity (phase 1). Following the abrupt change in SRP concentration, a quasi-linear increase continued for at least 1 day (phase 2). Because the rate of increase of the SRP concentration was proportional to the transfer rate as shown in Eq. (1), phase 1 corresponded to a transient state in which the SRP transfer rate changed from an abrupt transfer to a steady transfer, and phase 2 denoted a steady release that continued for more than 1 day.

\subsection{Model-based calculation results}

Figure 3a shows the response of the SRP concentration profile to the step change in the flow velocity from $1.7 \mathrm{~cm}$ $\mathrm{s}^{-1}\left(u^{*}=0.08 \mathrm{cms}^{-1}\right)$ to $5.0 \mathrm{~cm} \mathrm{~s}^{-1}\left(u^{*}=0.20 \mathrm{~cm} \mathrm{~s}^{-1}\right)$, as simulated by the non-steady-state numerical model described earlier. Figure $3 \mathrm{~b}$ also shows the variation in the SRP profile in response to the step change in the flow velocity from 5.0 to $10.0 \mathrm{cms}^{-1}$. Figure 3 shows that the DBL thickness decreased following the increase in the flow

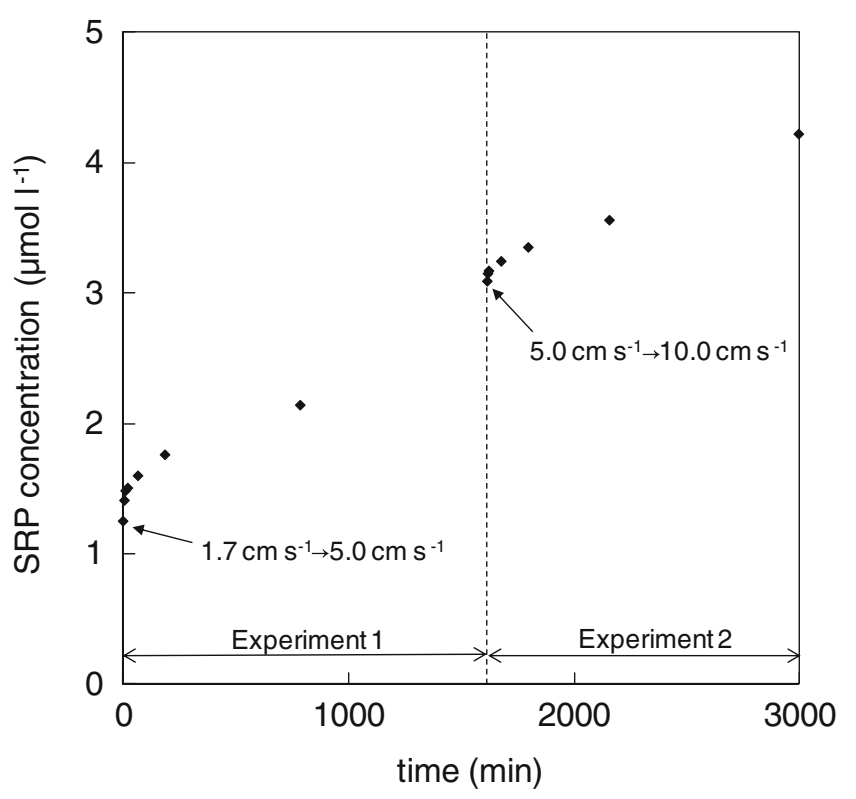

Fig. 2 Time series of soluble reactive phosphorus (SRP) concentration in the overlying water during the experiment. A rapid increase in the SRP concentration was observed for approximately $5 \mathrm{~min}$ after the step change in the flow velocity. Following the abrupt change in concentration, a quasi-linear increase continued for at least 1 day 

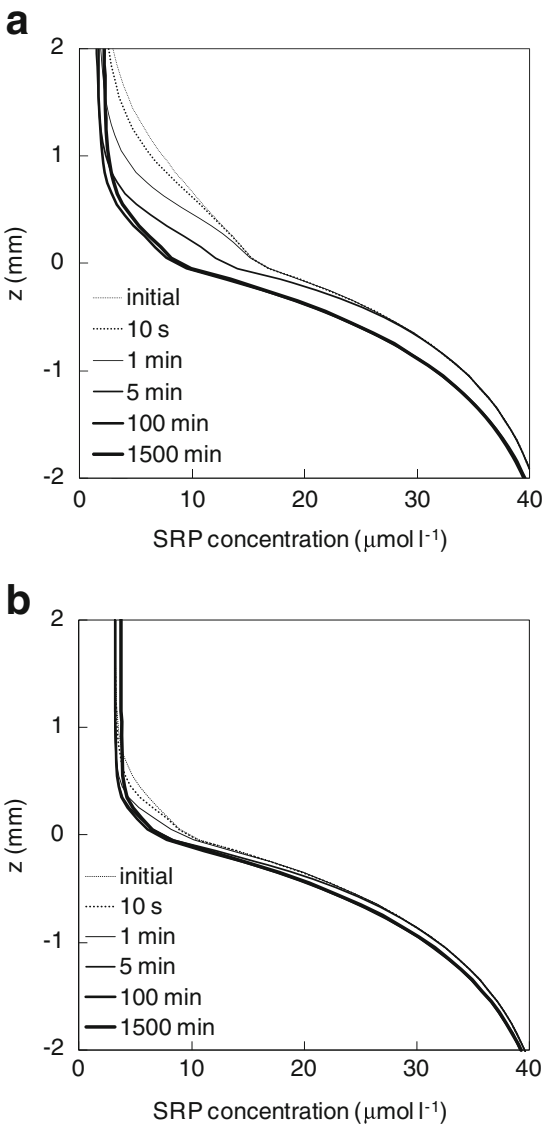

Fig. 3 Variation in soluble reactive phosphorus $(S R P)$ concentration profiles after a step increase in flow velocity: a from 1.7 to $5.0 \mathrm{cms}^{-1}$, b from 5.0 to $10.0 \mathrm{cms}^{-1}$

velocity. This phenomenon is common and implies that the SRP that was contained in the former DBL was transferred to the upper water layer as a result of the increase in the flow velocity. Although the SRP concentration profiles in the overlying water almost attained a quasi-steady state within 5 min, Fig. 3 also shows that approximately 100 min was required to attain a quasi-steady state in the sediment. This is because the time scale of the biochemical reaction in the sediment is greater than that of diffusion in water (Inoue et al. 2000), and this relationship will be discussed later.

\subsection{SRP transfer after the step increase in flow velocity}

Figure $4 a, b$ shows the trends of the SRP transfer rate that were obtained from the experimental results after the step increase in flow velocity from 1.7 to $5.0 \mathrm{~cm} \mathrm{~s}^{-1}$ and from 5.0 to $10.0 \mathrm{~cm} \mathrm{~s}^{-1}$, respectively. Figure 4 also shows the results of the model-based calculation of the response of the diffusive fluxes of SRP at $z=0 \mathrm{~mm}$ and $z=10 \mathrm{~mm}$ under the same calculation conditions as in Fig. 3. Both the experimental and model-based calculation results for $z=10 \mathrm{~mm}$ demonstrate that the step increase in flow velocity induced an abrupt enhancement of the SRP transfer rate that continued a

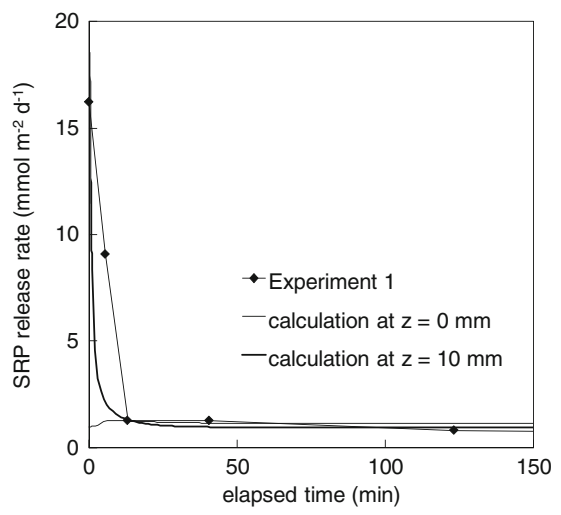

b

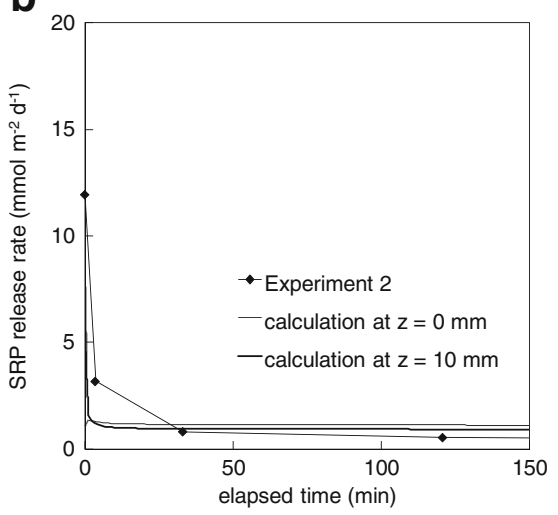

Fig. 4 Response of the diffusive soluble reactive phosphorus (SRP) transfers after a step increase in flow velocity: a from 1.7 to $5.0 \mathrm{cms}^{-1}$, b from 5.0 to $10.0 \mathrm{cms}^{-1}$

for several minutes immediately after the change, before approaching a steady value. The time scale of the transient state in experiment 1 is longer than that in experiment 2 . Based on the calculated results, the time-integrated diffusive transfer rate at $z=10 \mathrm{~mm}$ for the first $10 \mathrm{~min}$ after the step change was $2.99 \times 10^{-2} \mathrm{mmolm}^{-2}$, which was almost equivalent to diffusive transport for $46 \mathrm{~min}$ in the quasi-steadystate for experiment 1 . For experiment 2 , the time-integrated diffusive transfer rate at $z=10 \mathrm{~mm}$ for the first $10 \mathrm{~min}$ after the step change was $1.10 \times 10^{-2} \mathrm{mmolm}^{-2}$, which was almost equivalent to the diffusive transport for $17 \mathrm{~min}$ in the quasi-steady state. These results show the importance of considering the transient state and clearly demonstrate that the estimation based on a steady-state analysis underestimates benthic SRP transfer.

Figure 4 shows that the calculated diffusive transfer rates at the sediment-water interface $(z=0 \mathrm{~mm})$ had maximum values at 9 and $1 \mathrm{~min}$ after the step change in the flow velocity in experiments 1 and 2, respectively. These values are approximately 1.14 and 1.21 times larger than the values in the steady state $(t \rightarrow \infty)$ in experiments 1 and 2, respectively. These temporal maximums arise because the response of the SRP concentration profile in the DBL is 
much faster than that in the sediment, and thus the concentration gradient at the sediment-water interface becomes temporarily steep. As shown in Fig. 3, and by the differences in the SRP transfer rates between $z=0 \mathrm{~mm}$ and $z=10 \mathrm{~mm}$, the significant increase in diffusive SRP transfer to the upper water layer results from the rapid transport of SRP that had accumulated within the DBL and from the intensified diffusion following a decrease in DBL thickness.

\section{Discussion}

\subsection{Effect of nitrate on the SRP transfer rate}

Here we summarize the results that were obtained in phase 2. The SRP release rate during the quasi-steady state of experiment $2\left(0.44 \mathrm{mmolm}^{-2} \mathrm{day}^{-1}\right)$ was smaller than that of experiment $1\left(0.57 \mathrm{mmolm}^{-2}\right.$ day $\left.^{-1}\right)$, i.e. the SRP transfer rate decreased when the mean flow velocity increased. According to the numerical model, the SRP desorption capacity in the sediment $\left(0.72 \mathrm{mmolm}^{-2}\right.$ day $\left.^{-1}\right)$ was large enough to compensate for the released SRP. Therefore, the SRP decrease in the pore water may not be the cause of the decrease in the SRP transfer rate in experiment 2.

Although the benthic nitrate transfer rate was, unfortunately, not measured in the laboratory experiment, the theoretical model-based calculations demonstrated that the nitrate transfer rate was an increasing function of flow velocity owing to the decrease in DBL thickness. Consequently, the enhanced nitrate supply resulting from the increasing flow velocity made the sediment surface layer more oxic (Andersen 1982) and suppressed SRP release from the sediment. This suppressive effect of nitrate on the release of SRP from the sediment has been reported previously, and the nitrate concentration that was measured in this experiment $\left(201.4 \mu \mathrm{moll}^{-1}\right)$ was high enough to suppress SRP release from the sediment (Boström et al. 1988). The oxic layer, which has a brown colour, was visible on the surface of the sediment, although the oxygen that was measured downstream of the flume was completely depleted throughout the experiment. Therefore, the oxidation-reduction potential in the surface layer of the sediment was not low.

\subsection{Time scale of SRP transfer fluctuations}

The time scale of diffusion in the DBL, $\tau_{\mathrm{d}}$, was defined as follows:

$\tau_{\mathrm{d}}=\frac{\delta^{2}}{2 D_{z}}$

where $\delta$ is the DBL thickness that was obtained by the concentration gradient at the sediment-water interface and the concentration in the bulk of the water and $D_{z}$ is the average diffusion coefficient in the DBL. From the calculated values, $\tau_{\mathrm{d}}$ was estimated to be small: $89 \mathrm{~s}$ in experiment 1 and $22 \mathrm{~s}$ in experiment 2. The abrupt increase in the SRP transfer rate resulting from the step increase in flow velocity is attributed to the short-time-scale response of the SRP concentration profile in the DBL.

Because the majority of the sediment was anoxic in the experiment, SRP desorption and diffusion in the sediment were the controlling processes for the fluctuation in SRP release from the sediment. The time scales of SRP desorption, $\tau_{\mathrm{de}}$, and SRP diffusion, $\tau_{\mathrm{s}}$, in the sediment are defined as follows (Inoue et al. 2000):

$\tau_{\mathrm{de}}=\frac{1}{k_{\mathrm{de}}}$

$\tau_{\mathrm{s}}=\frac{\lambda^{2}}{2 D_{z \mathrm{SP}}}$

where $\lambda$ is the length scale of the SRP concentration profile in the sediment that was obtained from the concentration gradient at the sediment-water interface and the concentration in the bulk of the sediment. In this study, we used $2.10 \times 10^{-4} \mathrm{~s}^{-1}$ for $k_{\text {de }}$, which yielded $\tau_{\mathrm{de}}=79 \mathrm{~min}$. On the other hand, because $\lambda$ was calculated to be $0.73 \mathrm{~mm}$ for experiment 1 and $0.92 \mathrm{~mm}$ for experiment 2 , the $\tau_{\mathrm{s}}$ values were $17 \mathrm{~min}$ for experiment 1 and $27 \mathrm{~min}$ for experiment 2. The calculated time variations in the SRP release rate at $z=0 \mathrm{~mm}$ after the step increase in flow velocity were well expressed by combinations of these phenomena. Moreover, as the experiment was conducted under anoxic conditions, the most important process for SRP release was SRP desorption. The time scale of SRP desorption $(79 \mathrm{~min})$ is much longer than that of the release rate fluctuation (less than $5 \mathrm{~min}$ ). Therefore, changing the value of $k_{\mathrm{de}}$ would have no overall consequences for the main findings.

\subsection{Other concerns}

A rapid increase in the SRP release rate was observed within $5 \mathrm{~min}$ after a step increase in flow velocity. During this period, the concentration in the bulk water increased by $0.15 \mu \mathrm{moll}^{-1}$ on average. By contrast, the measured SRP concentration in the pore water of the surface layer (i.e. $0-1 \mathrm{~cm}$ in depth) was $35.5 \mu \mathrm{moll}^{-1}$. Based on these measured values, the decrease in the concentration gradient between the water and the sediment was calculated to be $0.03 \mu \mathrm{moll}^{-1} \mathrm{~mm}^{-1}$, which is equivalent to approximately $0.4 \%$ of the initial gradient. Therefore, the effect of SRP accumulation on the concentration gradient and the benthic flux was considered to be negligible. 
Fig. 5 Schematic representation of non-steadystate variation in a vertical soluble reactive phosphorus profile in the vicinity of the sediment-water interface.

Dotted grey-coloured lines show the respective profiles during the previous stage: a before the step increase in flow velocity, $\mathbf{b}$ immediately after the step increase in flow velocity, $\mathbf{c}$ under a quasisteady state

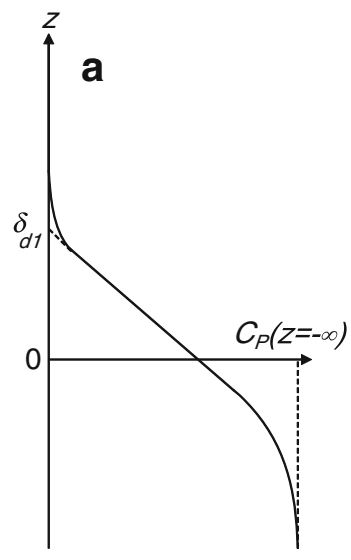

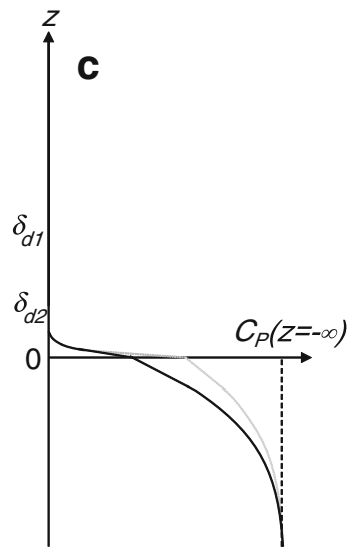

Regarding the enhancement factor, $F$, defined by Eq. (10), a $10-\%$ error in the friction velocity will cause ca. $0.26 \%$ error in $F$. Therefore, we concluded that changes in the friction velocity within a reasonable range would not affect our main findings.

\section{Conclusions}

Laboratory experiments with a rectangular closed flume were conducted to study the response of SRP transfer rates to a step change in flow velocity. A dynamic numerical model that predicted the non-steady-state variations in the SRP release rate was also developed, and calculations from this model were compared with the experimental results. Both sets of results showed a drastic increase in the SRP transfer rate within approximately 5 min of a step increase in the flow velocity in the overlying water. This temporal increase in SRP transfer was due to the rapid transport of SRP that had accumulated within the DBL to the upper water layer and was due to the intensified diffusion. Diffusive transfers in the overlying water reached a steady state within approximately $5 \mathrm{~min}$ because these transfers are mainly governed by the diffusion process in the DBL, which has a rather short time scale. The slower change in the SRP concentration profiles in the sediment was due to the long time scales of diffusive transport and/or biochemical reactions in the sediment. These differences in time scale caused the temporal maximum of the SRP gradient and transfer rate at $z=0 \mathrm{~mm}$ to occur after the increase in flow velocity. Figure 5 shows a schematic representation of the non-steady-state variation in a vertical $P$ profile in the vicinity of the sediment-water interface: (a) before the increase in flow velocity, (b) immediately after the increase in flow velocity and (c) in the steady phase after the increase in flow velocity. The variation in the vertical SRP profile before and after the step increase in flow velocity is explained by the following:

1. Before the step increase, the DBL is fully developed. The SRP concentration gradient at the sediment-water interface is gentle and steady (see Fig. 5a)

2. Immediately after the step increase in flow velocity, the DBL thickness decreases, and the SRP that has accumulated in the former DBL is transferred to the upper water layer by the intensified diffusion. Moreover, the thinning of the DBL causes a temporal increase in the concentration gradient and the diffusive transfer rate at the sediment-water interface, and this process continues for the diffusive time scale, $\tau_{\mathrm{d}}$ (see Fig. 5b).

3. Subsequently, the SRP concentration profile in the sediment approaches a steady state (see Fig. 5c).

Based on the experimental results and assuming that the bottom layer thickness is $50 \mathrm{~cm}$, which is typical of Lake Shinji, the increased SRP transfer in the first $100 \mathrm{~min}$, which is the required time for the SRP profile to stabilize, induces a $0.34-\mu \mathrm{moll}^{-1}$ increase in the SRP concentration in the bottom water layer. We consider this effect substantial and worthy of greater attention. In addition, this study also demonstrates the importance of accumulated materials in the DBL, not in the sediment or pore water. The increase in the diffusive transfer of nitrate following the velocity increase suppresses the SRP release rate from the sediment. The suppression of the SRP release rate occurs because the nitrate that is transferred to the sediment surface prevents the reduction of ferric hydroxide and SRP desorption. The model-based calculated results for the SRP transfer rate were in agreement with the experimental results. Therefore, our model can potentially simulate the response of the SRP concentration profile to temporal variations in flow velocity. 
Open Access This article is distributed under the terms of the Creative Commons Attribution License which permits any use, distribution, and reproduction in any medium, provided the original author (s) and the source are credited.

\section{References}

Andersen JM (1982) Effect of nitrate concentration in lake water on phosphate release from the sediment. Wat Res 16:1119-1126

Belanger T (1981) Benthic oxygen demand in Lake Apopka, Florida. Wat Res 15:267-274

Beutel MW (2006) Inhibition of ammonia release from anoxic profundal sediments in lakes using hypolimnetic oxygenation. Ecol Eng 28:271-279

Beutel MW, Leonard TM, Dent SR, Moore BC (2008) Effects of aerobic and anaerobic conditions on $\mathrm{P}, \mathrm{N}, \mathrm{Fe}, \mathrm{Mn}$, and $\mathrm{Hg}$ accumulation in waters overlaying profundal sediments of an oligomesotrophic lake. Wat Res 42:1953-1962

Boers PCM, Van Raaphorst W, Van der Molen DT (1998) Phosphorus retention in sediments. Wat Sci Tech 37:31-39

Boström B, Andersen JM, Fleischer S, Jansson M (1988) Exchange of phosphorus across the sediment-water interface. Hydrobiologia 170:229-244

Boudreau BP (1997) Constitutive equations. In: Diagenetic models and their implementation. Springer, New York, pp 91-164

Boudreau BP (2001) Solute transport above the sediment-water interface. In: Boudreau BP, Jørgensen BB (eds) The benthic boundary layer: transport processes and biogeochemistry. Oxford University Press, New York, pp 104-126

Boynton WR, Kemp WM, Osborne CG, Kaumeyer KR, Jenkins MC (1981) Influence of water circulation rate on in situ measurements of benthic community respiration. Mar Biol 65:185-190

Brand A, Dinkel C, Wehrli B (2009) Influence of the diffusive boundary layer on solute dynamics in the sediments of a seiche-driven lake: a model study. J Geophys Res: Biogeosci 114:G01010

Clesceri LS, Greenberg AE, Eaton AD (1998) Standard methods for the examination of water and wastewater. APHA, AWWA, and WEF, Maryland

Fowler B, Drake J, Hemenway D, Heaney SI (1987) An inexpensive water circulation system for studies of chemical exchange using intact sediment cores. Freshwat Biol 17:509-511

Fujita K, Lee I-C, Nakashima M, Kumamoto M, Kusuda T (1997) Eutrophication in Hakata Bay (part 1) - seasonal variations of water and sediment quality. Environ Eng Res 34:231-238

Gächter R, Meyer JS, Mares A (1988) Contribution of bacteria to release and fixation of phosphorus in lake sediments. Limnol Oceanogr 33:1542-1558

Gomez E, Durillon C, Rofes G, Picot B (1999) Phosphate adsorption and release from sediments of brackish lagoons: $\mathrm{pH}, \mathrm{O}_{2}$ and loading influence. Wat Res 33:2437-2447
Gonsiorczyk T, Casper P, Koschel R (2001) Mechanisms of phosphorus release from the bottom sediment of the oligotrophic Lake Stechlin: importance of the permanently oxic sediment surface. Arch Hydrobiol 151:203-219

Gunnars A, Blomqvist S (1997) Phosphate exchange across the sediment-water interface when shifting from anoxic to oxic conditions - an experimental comparison of freshwater and brackishmarine systems. Biogeochem 37:203-226

Hosoi Y, Murakami H, Kozuki Y (1992) Oxygen consumption by bottom sediment. Proc JSCE, Hydraul Sanit Eng 456(II):8392

Inoue T, Nakamura Y (2002) Diel variation of resuspension and effect of resuspension on phosphate release rate from the sediment. In: Ninokata $\mathrm{H}$, Wada A, Tanaka N (eds) Advances in fluid modeling and turbulence measurements. World Scientific, Singapore, pp 801-808

Inoue T, Nakamura Y (2009) Effects of hydrodynamic conditions on sediment oxygen demand: experimental study based on three methods. J Environ Eng 135:1161-1170

Inoue T, Nakamura Y (2011) Effects of hydrodynamic conditions on DO transfer at a rough sediment surface. J Environ Eng 137:2837

Inoue T, Nakamura Y, Adachi Y (2000) Non-steady variations of SOD and phosphate release rate due to changes in the quality of the overlying water. Wat Sci Tech 42:265-272

Jørgensen BB, Marais DJD (1990) The diffusive boundary layer of sediments: oxygen microgradients over a microbial mat. Limnol Oceanogr 35:1343-1355

Löfgren S, Böstrom B (1989) Interstitial water concentrations of phosphorus, iron and manganese in a shallow, eutrophic Swedish lake-implications for phosphorus cycling. Wat Res 23:1115-1125

McQueen DJ, Lean DRS, Charlton MN (1986) The effects of hypolimnetic aeration on iron-phosphorus interactions. Wat Res 20:1129-1135

Nakamura Y (1994) Effect of flow velocity on phosphate release from sediment. Wat Sci Tech 30:263-272

Nakamura Y, Mikogami M (1994) Near-bed turbulence and mass transfer at the sediment-water interface. Ann J Hydraul Eng JSCE $38: 223-228$

Sørensen J (1982) Reduction of ferric iron in anaerobic, marine sediment and interaction with reduction of nitrate and sulfate. Appl Environ Microbiol 43:319-324

Spears BM, Carvalho L, Perkins R, Kirika A, Paterson DM (2007) Sediment phosphorus cycling in a large shallow lake: spatiotemporal variation in phosphorus pools and release. Hydrobiologia $584: 37-48$

Ullman WJ, Aller RC (1982) Diffusion coefficients in nearshore marine sediments. Limnol Oceanogr 27:552-556

Urban-Malinga B, Opalinski KW (1999) Vertical zonation of the total, biotic and abiotic oxygen consumption on a Baltic sandy beach. Oceanol Stud 28:85-96 